\title{
Grand Challenges in Engineering Project Organization
}

\author{
Vivek Sakhrani ${ }^{1 *}$, Paul S. Chinowsky ${ }^{2}$, and John E. Taylor ${ }^{3}$
}

\begin{abstract}
Grand Challenges are ambitious yet specific goals to galvanize a scholarly community for focused research and high impact. We solicited contributions from ten leading thinkers in the field of Engineering Project Organization (EPO) to elicit their visions for EPO research. Based on content analysis of the text in these statements, and analytical concept mapping, we created a Grand Challenges synthesis framework to set the stage for the future of EPO research. This technical paper describes the six Grand Challenges and associated research thrusts that could define the future of scholarship and impact in the field. The paper also reflects on recent contributions to the field and identifies both barriers and community aspirations to addressing the Grand Challenges.
\end{abstract}

\section{Keywords}

Grand Challenges, infrastructure, projects, systems, engineering, organization

\footnotetext{
1 Massachusetts Institute of Technology and CPCS Transcom Inc., 1050 Connecticut Avenue NW Suite 500, Washington DC 20036,*Corresponding Author: E-mail: sakhrani@alum.mit.edu

${ }^{2}$ Department of Civil, Environmental and Architectural Engineering, University of Colorado Boulder

${ }^{3}$ School of Civil and Environmental Engineering, Georgia Institute of Technology
} 


\section{Introduction}

The Engineering Project Organization (EPO) community has become increasingly prolific, publishing in both this journal as well as other disciplinary and topical forums. The range of topics remains diverse, covering project design, delivery, management, and governance. Research methods both established and exploratory - continue to provide new insights. After over a decade of organizing a community of scholars in EPO, the Engineering Project Organization Society embarked on a process of reflection to channel our efforts as a community to guide the choice of research topics and applicable methods for future research. Over the last decade, this dialogue has been explored and built upon at the annual Engineering Project Organization Conference (EPOC), which convenes scholars and practitioners.

The 2014 Conference featured a 'Grand Challenges' panel as a forum for taking stock and to envision the future of EPO research. In preparation for this panel, we invited ten leading thinkers to describe what they foresaw as the field's Grand Challenges (GCs). Each of the invited contributors shared brief Grand Challenge essays in advance of the conference. We refer to these essays as GC Vision Statements. Four of the ten contributors commented on the body of essays during a panel held at the conference.

Over the last three years, these GCs have formed the basis of multiple discussions and research efforts throughout the community. The trials of addressing a Grand Challenge emerge in full scope once a group attempts to move from awareness to action. We revisit these GCs in this paper, including an overview of the original identification and definition of the Challenge, the responses to the Challenges, and an analysis of where we are at the present time. We evaluate recent community contributions from the EPOC 2017 conference and take stock and identify barriers to addressing the Grand Challenges. The paper builds upon this review and reflection process to once again charge the community with a renewed focus on achieving progress on the Grand Challenge issues.

\section{Concept: Grand Challenges}

Communities often use the term Grand Challenges to set goals or targets for their efforts, based on a process of focused discourse. We look to some established definitions and examples to contextualize what we mean by GCs and the importance of the community's role in developing them. An understanding of the attributes of GCs can help us intentionally frame our own for the EPO community.

The White House Office of Science and Technology Policy (OSTP) defines Grand Challenges as "....ambitious but achievable goals that harness science, technology, and innovation to solve important national or global problems and that have the potential to capture the public's imagination" (OSTP 2013). While the White House definition suggests a distinct policy context, it encapsulates ideas that are broadly relevant to scholars as well as practitioners. GCs are therefore specific enough that they can be both ambitious yet achievable. Further, the framing of GCs may point to specific mechanisms ("harness..."). GCs also provide a compelling sense of impact, justifying the effort and resources necessary to address them. In a policy context, one specific type of impact is to capture the public imagination to secure the necessary support and resources for pursuing GCs.

Examples of GCs that are more community focused include the Hilbert problems in mathematics, one of the earliest modern incarnations of goal setting for an entire scholarly field (Hilbert 1900). In the late $19^{\text {th }}$ century, the German mathematician collected and laid out 23 problems in mathematics, including the Riemann Hypothesis. Hilbert's effort inspired generations of mathematicians to attempt to solve these problems (Giles 2011). In the interdisciplinary engineering field, the National Science Foundation and the National Research Council convened an international committee to develop a list of Engineering Grand Challenges for the $21^{\text {st }}$ century, which Charles Vest, then President of the National Academies of Engineering, described as "visionary, terribly important, and do-able" (Vest 2008). The Bill \& Melinda Gates Foundation published a list of fourteen GCs for global health in 


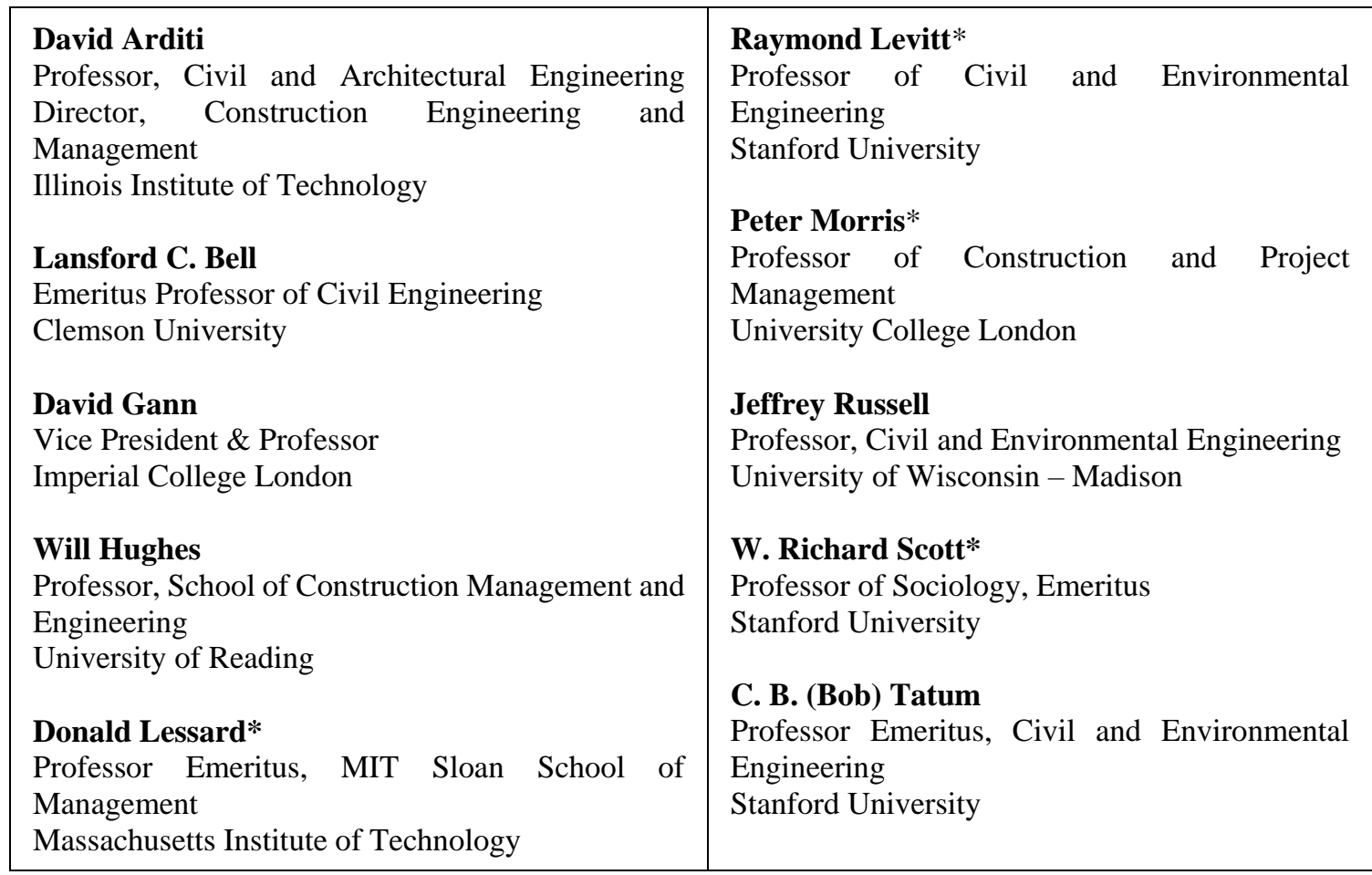

Table 1. Grand Challenge Vision Statement Contributors

(Names and Affiliations as of EPOC 2014; * indicates GC Panelist)

2003. These were fashioned on the basis of a number of definitions specific to healthcare: "[GCs are] specific scientific or technological innovations that would remove a critical barrier to solving an important health problem in the developing world with a high likelihood of global impact and feasibility" (Varmus et al. 2003) and later "[a GC is] a specific critical barrier that if removed would help to solve an important health problem" (Daar et al. 2007). More recently, the social sciences have also articulated a set of ten challenges (Giles 2011).

Grand Challenges therefore have the following key characteristics: (i) articulated by stakeholders, (ii) specific (ii) ambitious yet feasible (iii) framed in a manner that suggests the use of specific methods or disciplines, and (iv) have the potential for broad impact. The GCs for EPO developed later in the paper embody these attributes. Not only are the GCs important goals to galvanize the research community, but the discursive process for identifying GCs also engages the community.

\section{Contributors}

Following this rationale, we engaged leading thinkers in the EPO community to respond to the open-ended prompt: "Please describe your vision for research grand challenges in Engineering Project Organization." No other guidelines were set down, and no restrictions were placed on content, structure, or format of responses. The ten leading thinkers we invited have each had illustrious careers in their respective fields, which intersect in the topic and areas of the field of EPO. Table 1 lists the names and affiliations of the contributors at the time of the EPOC 2014 conference. Each scholar had achieved a title of full professor and all have had leadership roles at their institutions or in their scholarly communities or both. Their departmental affiliations cover civil engineering, environmental engineering, construction engineering, sociology, management, strategy, and innovation. Four of the contributors 


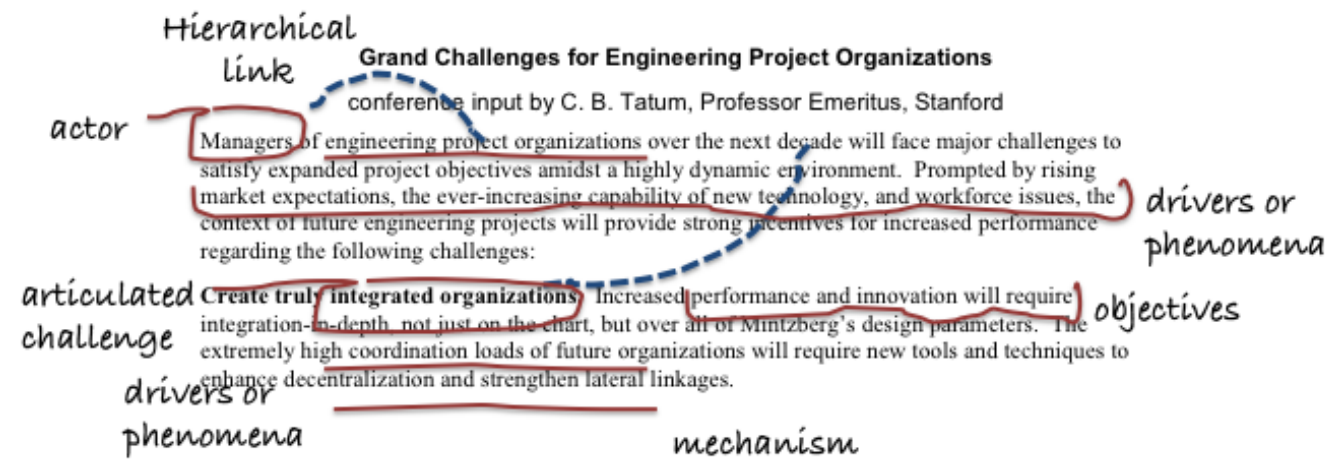

Figure 1. Content analysis coding example for eliciting Grand Challenges from Vision Statements

were able to participate as GC panelists at the EPOC 2014 conference. They reviewed contributions by the other panelists and presented holistic views of the GCs, which is documented in the synthesis that follows.

\section{Method}

We primarily used content analysis to study the Grand Challenge Vision Statements. Content analysis is a family of analytical, qualitative research approaches used to interpret meaning from text data (Weber 1990; Krippendorf 2012).

Content analysis served two purposes in our elicitation of GCs. First, it enabled a detailed scrutiny of the main concepts and ideas in each contributor's statement, and the categories emerged inductively. In this sense, our approach to content analysis was "conventional" as defined by Hsieh and Shannon (2005). Second, it allowed us to link the concepts and their categories across the submitted statements. In this fashion, we treated each statement as an individual stand-alone section of text as well as a summative body of text when combined.

We show an example of the coding process in Figure 1. Sections of text were coded phrase-byphrase and line-by-line to identify key concepts. In the first line, for example, manager is identified as an actor. The manager is embedded within an engineering project organization. This relationship is a hierarchical link denoting different units of analysis (i.e. an individual versus an organization).
Other concepts such as dynamic environments and technology capabilities also emerge. Possible category labels for these are drivers / phenomena. The next few phrases in the text contain examples of an articulated challenge, integrated organization, the potential impact stated as objectives, increased performance and innovation, and a mechanism for addressing the articulated challenge, decentralization. Through this section, we have thus identified some units of analysis and their hierarchical relationships. We have also noted conditions under a category of drivers and phenomena. Further, we have a specific challenge, and a mechanism for addressing it with some description of its potential impact. As we proceed through the text (and through other Vision Statements), we continue to refine these over the entire body of material.

We then created a concept map to collectively summarize the analysis of the ten Vision Statements. The main concepts in these categories are linked to each other based on content analysis, and the linkages provide a narrative flow and logical construction of research agendas. We discuss the synthesis in detail below.

Finally, we also evaluated recent contributions to the EPOC 2017 conference through content and keyword analysis, to take stock and compare progress towards addressing the synthesized Grand Challenges. The paper ends with our reflections on this progress and resulting aspirations. 


\section{Synthesis}

Two categories of concepts emerged from content analysis of the Vision Statements: 'Drivers' and 'Grand Challenges'. Drivers are summary or umbrella categories for different phenomena. Drivers create the conditions and needs, and set the stage for Grand Challenges as opportunities for research. Understanding the role of Drivers is therefore key to making the case for Grand Challenges.

\section{Drivers}

We identified a number of interacting phenomena in the bodies of text, and summarize them under Drivers, which are umbrella constructs that encapsulate related phenomena. The emergent Drivers we posit are the concepts of 'International Teams', 'Global Project Opportunities', 'Complexity and Uncertainty', 'Climate Change', and 'Social Well-being'.

Figure 2 uses International Teams as an example to show how we denoted the relationship between specific phenomena and the umbrella concept in the concept map. Members of International Teams have different nationalities and differ more widely in their sources of motivation, leadership styles, culture and legal perspectives, than the members of the same nationality in intra-country or domestic

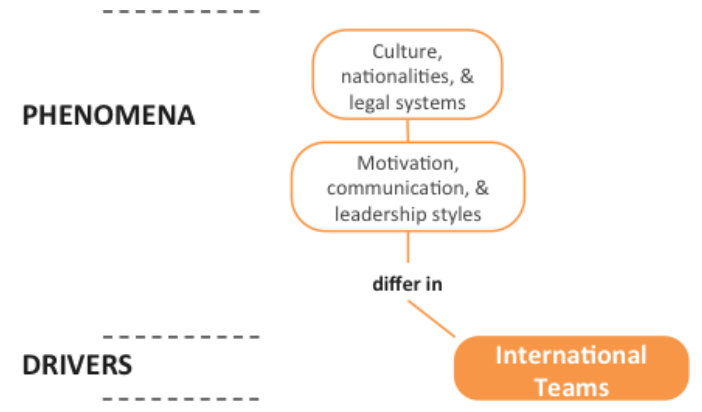

Figure 2. Example of the emergent 'International Teams' Driver, with the phenomena that influence it

teams (Goodman et al. 1999, Gundersen et al. 2012). Differences in these intrinsic attributes and resulting dynamics can affect work quality and project outcomes (Chi and Levitt 2011, Ramalingam et al. 2014).

A set of phenomena can contribute to more than one Driver, since the latter are umbrella concepts. Continuing with the International Teams example, other phenomena that influence this Driver include national priorities, locations and geography, and the role of emerging markets. Whereas both individuals and groups in teams reflect cultural influences and leadership styles, national priorities and geographic locations can be thought of as influencing the objectives and work conditions of

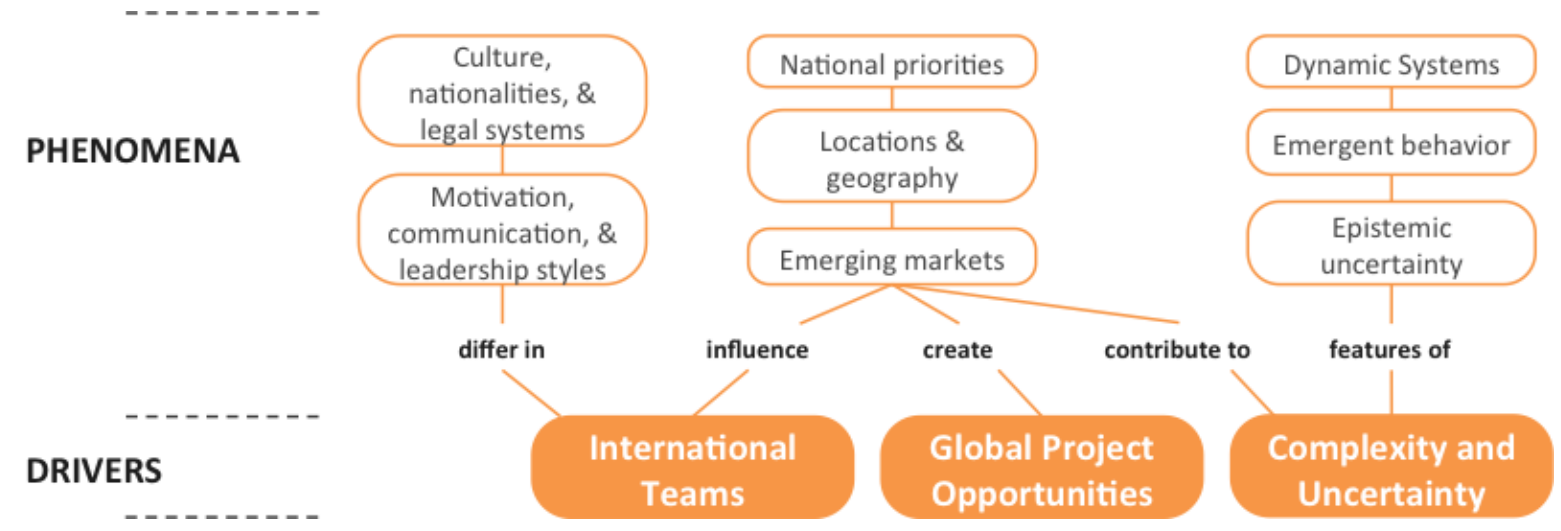

Figure 3. Phenomena could map to multiple Drivers 


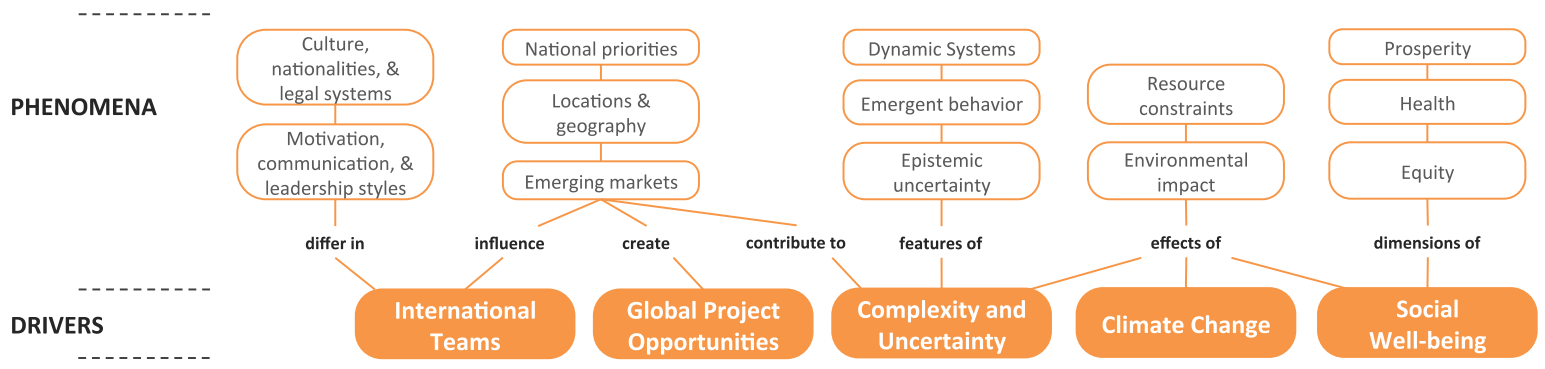

Figure 4. Full concept map of Phenomena and Drivers

those teams. However, these very same phenomena also create the conditions for Global Project Opportunities, a different Driver (see Figure 3). This latter umbrella concept points to the dynamics that motivate firms and sovereign sponsors to pursue projects in other countries to capture growth opportunities, to differentiate and partner in remote locations and geographies for untapped resources, (Garcia et al. 2014), or to further strategic sovereign and geopolitical interests (Zweig and Jinhai 2005, Haberly 2011, Scott et al. 2011). Taken together, these phenomena imply that many project opportunities are likely to continue to be global in nature, thus establishing a Driver for Grand Challenges.

At the same time, the phenomena that create Global Project Opportunities also contribute to Complexity and Uncertainty (also shown in Figure 3), a separate Driver that affects project structure, behavior and environments (Morris and Hough 1987, Baccarini 1996, Williams 2005, Lessard et al. 2014). This Driver in turn encapsulates some other features such as the phenomena of dynamic systems, emergent behavior, and epistemic uncertainty, to acknowledge the dynamic nature of risks and emergent project outcomes in complex projects.

The last two Drivers that emerged from content analysis are Climate Change and Social WellBeing. Climate Change recognizes the influence of resource constraints on projects (Jeuland et al. 2017) and the environmental impact of projects and changing nature of climate-induced project risks (Chinowsky et al. 2011), on built environment projects in particular. Social Well-Being acknowledges social dimensions of prosperity, health, and equity, and how they manifest in stakeholder and community values and conflicts (Kaminsky and Javernick-Will 2014, Lousberg et al. 2016). The phenomena related to Climate Change also influence Social Well-Being.

These last two umbrella concepts denote phenomena that have only recently attracted attention in the discourse on project outcomes and project management. While they have been previously studied in depth in other disciplines, the field of Engineering Project Organization is increasingly recognizing the relevance of these phenomena for projects research, and we posit that these should be considered as part of the mainstream of projects research.

Figure 4 shows the full mapping of phenomena identified in the Vision Statements and the five Drivers that emerged. These Drivers set the stage for the discussion of Grand Challenges.

\section{Grand Challenges (GCs)}

We synthesized five Grand Challenges out of the Vision Statements submitted by respondents. The GCs are 'the New Project Manager', 'Project Networks', 'Innovation and Growth Strategies', 'Systems Integration', and 'Lifecycle Value and Governance'. The unit of analysis for the first of these concepts, New Project Manager, is an individual and the level of analysis is intra-firm or intra-project and possibly within teams. For the last four on the other hand, the unit of analysis is typically the project, looking intra- or across firms, networks or systems. The last GC in particular, 
Lifecycle Value and Governance, uniquely focuses on the outcome dimensions of projects.

Similar to Drivers, the GCs are umbrella terms for a large number of specific research topics or thrusts. We discuss each GC concept and showcase both its related Drivers as well as the corresponding research topics that can advance the field of EPO. In the discussion below and each GC summary, the topics in bold are those identified as requiring further research to address the Grand Challenges.

\section{GC1: The New Project Manager}

This GC addresses the education, role, and training-based competencies of the individuals in project management roles, and also their functionbased leadership behaviors, boundary spanning efforts, and self-directed learning capabilities.

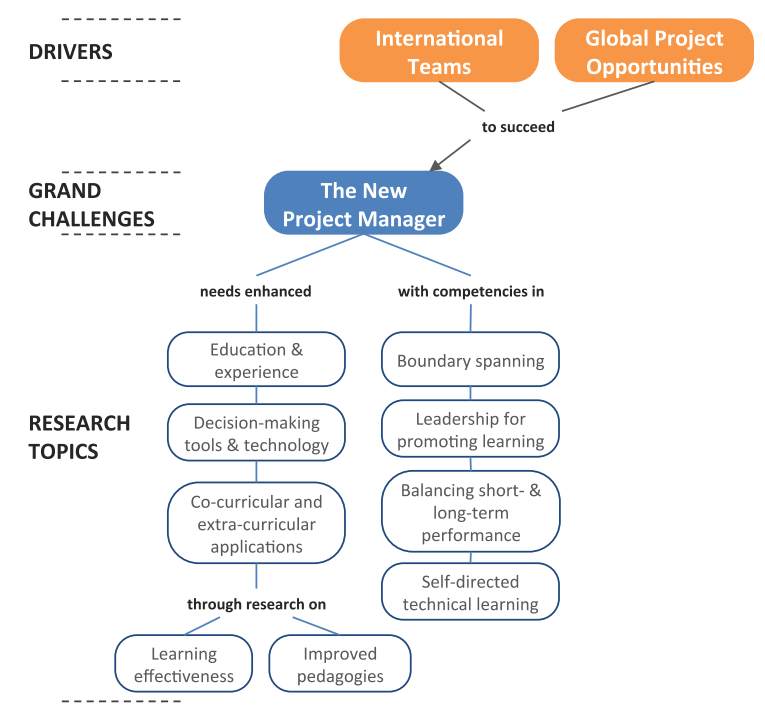

Figure 5. The New Project Manager Grand Challenge (GC1) with its Drivers and associated research topics.

Many respondents argued that the nature of projects and project management continues to evolve, whereas training and curriculum for project managers remains static and anchored to project approaches of a past era. One respondent articulated the need for new project management styles by describing the Drivers that relate to it
(International Teams and Global Project Opportunities):

"...the biggest challenge is the internationalization of engineering project organizations caused by the globalization movement, i.e., projects in different locations undertaken by teams composed of firms/personnel of different nationalities. As a result, project organizations are impacted by factors such as differences in culture, legal systems, priorities, diversity, and motivation/leadership styles of the different project participants... there will be more of these projects in the future."

Another statement reflected on the traditional education models that have been in place for decades:

"During my forty-five years in civil engineering university education the debates have remained the same. How much liberal arts education in the curriculum? Are business and finance courses important for engineers? Leadership and communication skills are important, but how do we teach that? Should we drop the thermodynamics course and replace it with something related to global cultural awareness?"

Yet other statements suggested the direction of research and pedagogy both within the classroom and beyond, captured in the sentiment of the following statement:

"...we need to focus on training the project managers of the future. No easy task. We need to continue to develop the tools that will make the project managers of the future more successful... Research is needed to determine just WHAT experiences outside the classroom are effective with respect to training our future project management professionals."

In the process of reflection, invited respondents thus laid out a number of topics on the education and training aspects of project managers as 
professionals that can help the growth of the New Project Manager, mapped in Figure 5.

This GC further provides a function- and behaviorbased perspective on the individual in the project manager's role (Gann et al. 2012). One respondent posed the question:

"... at the level of the individual...what style of leadership to balance short and long-term performance and a culture of learning, what type of competencies are required to span boundaries and deliver quality with rigor?"

GC1 Summary: In the context of International Teams and Global Project Opportunities, the New Project Manager needs enhanced education \& experience, decision-making tools \& technology, and co-curricular and extra-curricular applications, which will be developed through

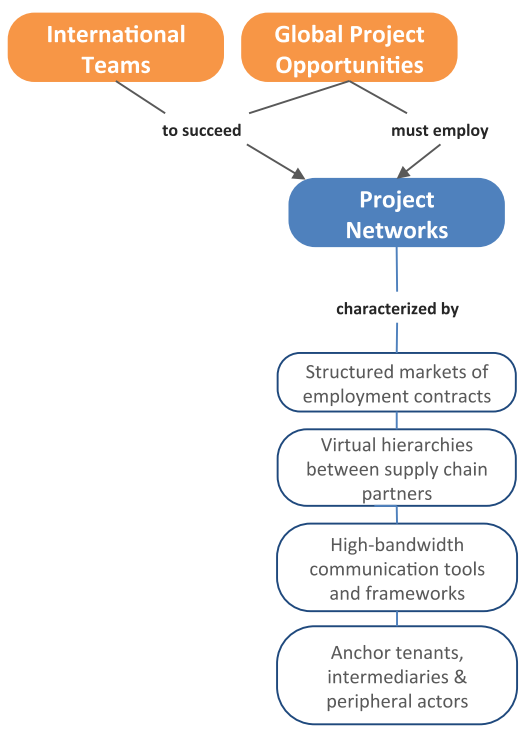

Figure 6. The 'Project Networks' Grand Challenge (GC2) with its Drivers and associated research topics.

research on learning effectiveness and improved pedagogies. The New Project Manager also spans technical and geographical boundaries, leads in a manner that promotes learning across the project organization, and balances short- and long-term performance in the project by taking a systems view. The individual also pursues self-directed technical learning to fulfill these functions.

\section{GC2: Project Networks}

This Grand Challenge is about framing projects that are driven by Global Project Opportunities, and comprised of International Teams. The frame involves the nature of contracts, supply chains, communication technologies and approaches, and the relationships between project actors and stakeholders (Figure 6).

One Vision Statement invoked Stinchcombe's (1985) view of project managers in large construction project networks, and posited a question:

"system integrators use contracts
between supply chain partners to create
elements of hierarchy in inter-firm
networks. What are the elements of
hierarchy and how should they be
implemented?"

On the topic of contracts and hierarchies, the statement further urged:

"Large construction projects should be re-conceptualized as organizational entities that have characteristics of both structured markets and virtual hierarchies: their organization and governance are formed by networks of employment contracts with direct-hire employees and various kinds of contracts with supply chain partners at the same and upstream/downstream stages of the value chain"

In response to the need for communication among different actors in these structured markets with contracts and hierarchies, one solution offered was to enable peer-to-peer communication of rich media between project participants by designing high-bandwidth communication networks, tools and frameworks to support sophisticated "publish and subscribe" protocols.

At the same time, another respondent added further nuance to the types of actors in Project Networks, delineating them as principal and peripheral: 
"...many of the skills required for successful projects are not lodged in any of the principal players - whether government agencies or private firmsbut in seemingly peripheral or marginal organizations - for example, in transaction advisors, advocacy associations, or public and nonpublic regulators. These intermediary organizations often provide background knowledge, expertise, negotiation and oversight skills that can be put to use by the central parties."

This Vision Statement posits that further research is needed on how peripheral actors can work in concert with principals to embed capacity in projects.

GC2 Summary: This Grand Challenge frames project organizations as Project Networks characterized by structured markets of employment contracts and virtual hierarchies in supply chains, needing high-bandwidth tools to facilitate communication between anchor tenants as principal actors, and other intermediaries and peripheral actors to leverage skills and build capacity.

\section{GC3: Innovation and Growth Strategies}

This GC focuses on the strategic aspects of Engineering Project Organization, and particularly on the need for innovation driven by Global Project Opportunities and the Complexity and Uncertainty that is a feature of projects and their environment. Strategies touch on organizational learning, knowledge management, and collaboration.

One Vision Statement described the need for innovation in EPO as follows:

"The challenge for EPO is how they innovate and grow. Incumbents may be too small to engage technical, managerial and financial capabilities required to deliver at scale: the sector does not exhibit concentration found in other industries."
Some respondents effectively connected the Drivers with prevalent business models in the argument for new strategies and innovation:

"How are we to construct the kind of commercial deals that motivate behaviors that may move us incrementally towards a better solution? What kind of contracts, guarantees and payment regimes will help to move the construction and engineering sectors from Victorian ideas of business into a modern business sector that encourages and rewards investment and innovation that benefits more than a privileged minority."

An initial set of questions was posited to address this Grand Challenge, covering resources, learning, and firm-level capacity and adaptation:

"How is growth resourced? How do firms develop long-term options to provide resilience and the capacity to adapt to future market, technological and systems uncertainty? What routines can be developed and embedded to foster learning from project to project, within EPO and across the sector?"

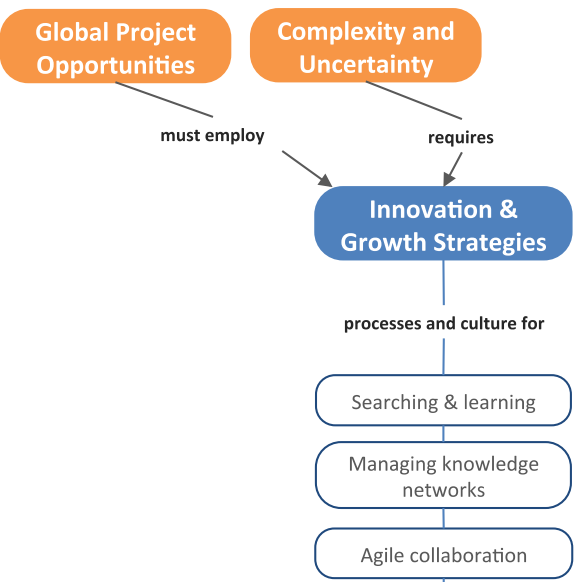

Figure 7. The 'Innovation and Growth Strategies' Grand Challenge (GC3) with its Drivers and associated research topics. 
A possible solution offered touched on the use of agility to help create shared knowledge:

"Enable and incentivize (by appropriate contracts and communications bandwidth) and encourage (by a supportive project culture and incentives) the development of 'shared awareness' of rapidly changing situations by all project participants, and 'selfsynchronization' among supply chain partners in which they share their knowledge and other resources autonomously and flexibly as the need arises"

GC3 Summary: This GC highlights the need for strategic choices to support innovation, by focusing on business models and mechanisms to enable a culture of searching and learning, managing knowledge networks, and agile collaboration.

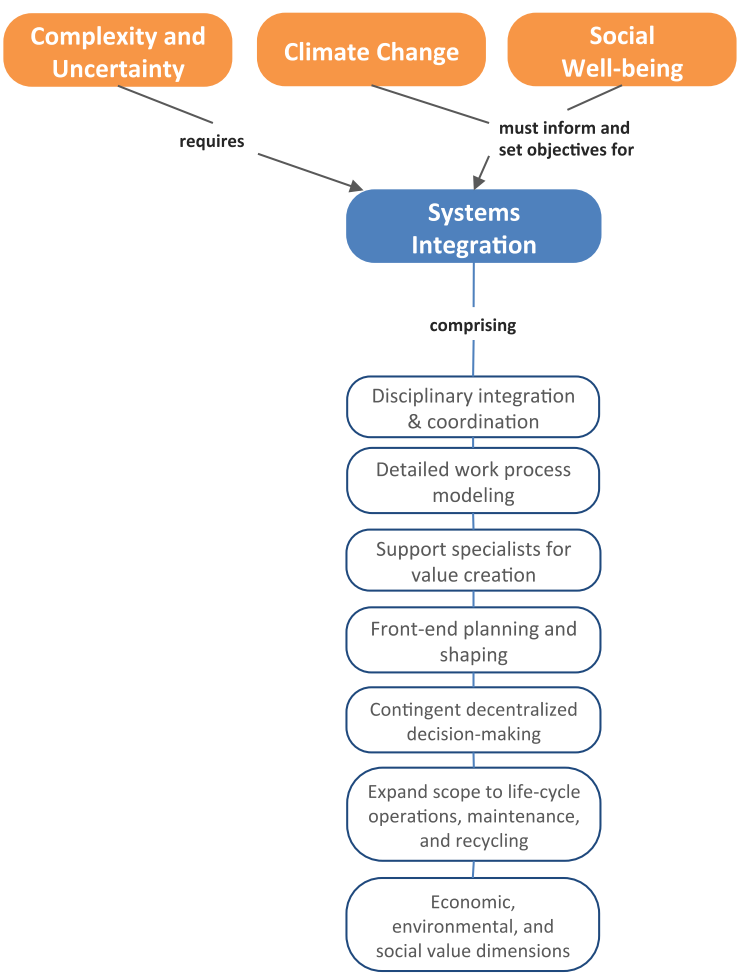

Figure 8. The 'Systems Integration' Grand Challenge (GC4) with its Drivers and associated research topics.

\section{GC4: Systems Integration}

The phenomena under the umbrellas of Complexity and Uncertainty, Climate Change, and Social WellBeing drive the fourth Grand Challenge of Systems Integration. The research topics under this GC include a number of issues in the areas of the disciplines of EPO, approaches to model and shape projects in the front-end, contingency approaches to decision-making, a systemic view of the project lifecycle to include operations, maintenance and recycling, and finally a broadening of the outcome or value dimensions of projects to include economic, environmental and social indicators.

We cannot overemphasize the degree to which the Drivers of Climate Change and Social Well-Being occupied the attention of respondents. Some offered sobering views of the nature of this challenge, both in terms of changing climate and its impact on society, and the direction of economies:

"It is increasingly difficult to deny that climate change is happening... The best we can hope for now is to mitigate the worst effects, and seek immediately to reduce those activities that contribute to climate change... the idea of continuous economic growth for everyone will soon become untenable... The twin challenge for our research community is in engineering solutions for mitigating climate change and contributing to the wider social debate on how the affluent parts of the world may make do with less."

In the words of one respondent, major projects will have to grapple with these issues from a systems point of view:

"The downside of this enormous growth in material output is the rapidly deteriorating physical environment and an unacceptable level of inequality and lack of social inclusion. The large challenge is how as a society we engage to increase wellbeing and not just GNP, taking into account system-level tradeoffs, and not become frozen into 
inaction. Many of these issues will have to be confronted within major projects."

From a systems integration and modeling perspective, one respondent called for broadening the outcome dimensions of value in projects, and exploring the possibilities of project design and work processes systematically to enhance value:

"Managing projects with inherent tradeoffs requires a focus on life cycle value measured in economic, social and environmental terms, certainly not just cost and schedule... This implies the need for more visibility into the drivers of value(s) and more opportunities for exploration of the design trade space, and more linkages at all levels to overall accountability, while at the same time modularizing activities to allow specialization, focus, and parallel work."

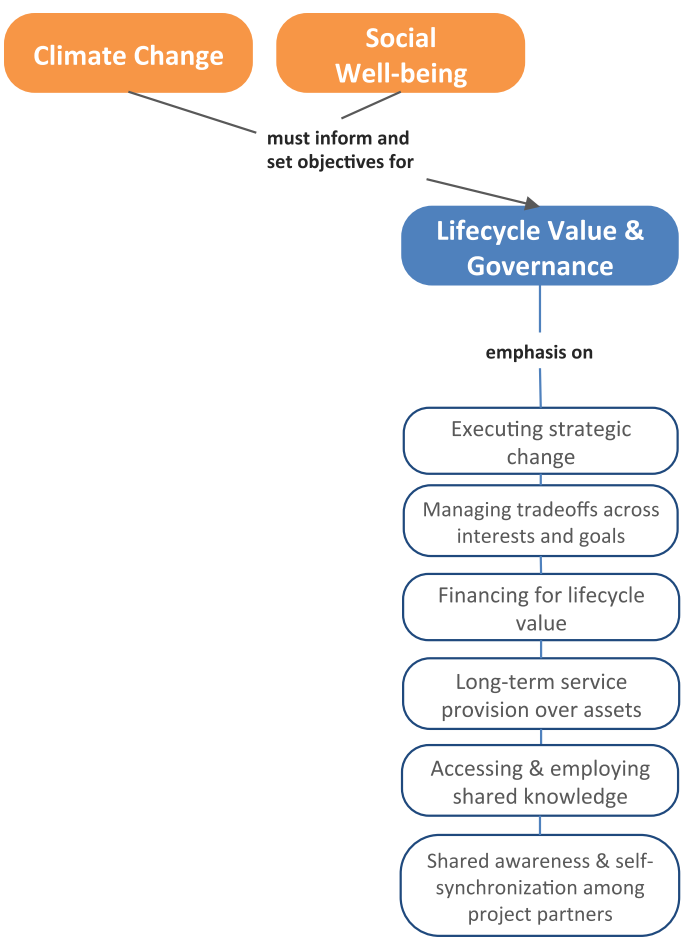

Figure 9. The 'Lifecycle Value \& Governance' Grand Challenge (GC5) with its Drivers and associated research topics.
The deeper dive into the project design space, integration of disciplines, and multi-dimensional value also implies pushing the notions of project management to encompass the complete project lifecycle:

“...project management should be more of spiral than a waterfall deeper into project life, while still maintaining temporal and resource discipline. This will be particularly true of the early stages of projects - shaping the opportunity, shaping the project, and reshaping the project. Since reshaping may take place at several points, multiobjective problem solving may have to be brought back, thus the organizational capacity for managing these tradeoffs must be kept alive."

Another research topic suggested was the need for improved decision-making with broader value dimensions in mind:
"contingent rules for decentralizing decision making about "Who?" "When?" and even "What?" to different degrees on different projects and in different companies ... Need enhanced lifecycle, triple-bottom-line metrics guide more sustainable long-term governance"

The challenge of Systems integration thus involves not just embedding "rigorously developed, risk assessments, contingencies and real options into early stage project processes", but also pushing "EPO theory, concepts, practices and tools forward into operations, maintenance and recycling."

GC4 Summary: The Systems Integration GC involves pushing the boundaries of EPO and deeper dives into modeling and work processes to add rigor to shaping in the front end, better integrate disciplines, capture the value available through specialization and contingent decentralized decision-making, and expand research scope further into the project lifecycle to cover operations, maintenance and recycling with a view to economic, environmental, and social dimensions of project value. 


\section{GC5: Lifecycle Value \& Governance}

This GC is once again driven by Climate Change and Social Well-Being, emphasizing the importance of the longer-horizon and time dependent phenomena such as the resource constraints that impinge upon projects, environmental considerations, as well as the broader consequences of projects for society including health, equity, and prosperity. The GC re-conceptualizes the notion of projects and management to include a system lifecycle and projects for the provision of services instead of assets. This brings into play a number of topics such as strategic change management, financing, knowledge sharing, and partner synchronization.

One of the Vision Statements described the need for a lifecycle view of projects in EPO research in the following way:

"My biggest fear is our myopic preoccupation with production and efficiency. As long as we focus on the optimum delivery methods for construction projects, we will miss some important challenges that have the potential to render most of our work irrelevant."

Another respondent articulated this through an agency theory lens, with the potential solution of reframing what a project delivers:

"Large-scale projects frequently involve "broken agency" with perverse incentives across lifecycle phases, as well as across disciplines and trades within a single phase of the lifecycle...Broken agency in projects can be overcome by delivering the outputs of projects as long-term services rather than as deliverable products to be handed off to clients upon implementation."

At a strategic level, the lifecycle view should help motivate decision-makers to focus on long-term success:

"Project organization and management defines a language, concepts and tools for executing strategic change of all kinds. So executives need to understand these concepts and tools, and to perform their roles as sponsors, decision makers and resource providers, in helping projects to implement their strategies succeed."

Another aspect of lifecycle governance is the financing of projects as vehicles for serviceprovision. Some argued that the relationship between financing and governance needs to be researched further:

"There is a need to understand how the financing of projects affects their governance, and vice versa -i.e., how the organization and governance of projects affects the kind of financing that they are potentially able to attract on favorable terms."

Recognizing that the projects evolve and are shaped over time, the issue of the capacity to make decisions, and solve problems a la 'real options' has implications for the governance mechanisms in EPO:

"Creative thinking about value(s) tradeoffs (should) continue deep into a project, especially since new ways to unlock them are likely to be discovered by iterating between concept and physical work... Since reshaping may take place at several points, multiobjective problem solving may have to be brought back, thus the organizational capacity for managing these tradeoffs must be kept alive."

A lifecycle conceptualization also means that project actors must re-configure both strategies and processes to access and manage knowledge for long-term performance:

"Shared knowledge is the "coin of the realm' of project integration and performance. Briefly this means: What do design and construction need to know about each other's work and about operation of the completed facility? Depending on the topic, this level of knowledge can vary from the most 
fundamental concepts to detailed models that implicitly assume certain levels of understanding and performance for specific disciplines and trades."

GC Summary: Lifecycle Value and Governance conceptualizes projects as service-provision mechanisms, for which strategic change, financing and governance, and decision-making capabilities for managing trade-offs must be studied further. It also requires a deeper understanding of knowledge sharing among actors and their ability to synchronize and work together over a long-time frame in a decentralized yet coordinated manner.

\section{Summary}

The relationships between all the synthesized Drivers and Grand Challenges, along with their related phenomena and research topics is shown in Figure 10.

With a focus on the individual, a number of respondents suggested the need for understanding the role of education, decision-making tools, communication and leadership in the evolution of the 'New Project Manager'. Some posit that these managers play the role of systems integrators as they work to realize Global Project Opportunities in International Teams. They create and employ 'Project Networks' to support 'Innovation and Growth Strategies' through boundary spanning and a balanced view of short- and long-term performance.

Respondents envision that firms and organizations participating in 'Project Networks' develop and exercise 'Innovation and Growth Strategies' to thrive in the context of 'Complexity and Uncertainty'. Anchor tenants bring different capabilities than do intermediaries, or peripheral interests. Through high-bandwidth communication, knowledge sharing, and agile collaboration, these actors work in a decentralized manner for eventual 'Systems Integration'.

Respondents converged on the need to pursue deep 'Systems Integration' with a view towards influencing emergent 'Life-cycle Value and
Governance'. They envision that detailed modeling for all processes in the project life-cycle, from the early front-end to late stage operations and maintenance is necessary for understanding lifecycle value using improved metrics along technical, environmental, economic and social dimensions. Supporting disciplinary specialization while using contingent rules for decentralized decision-making may also be important in keeping system-level tradeoffs alive.

Grand Challenge respondents believe that addressing Climate Change and enhancing Social Well-being will require project leaders to be poised for executing strategic change through long-term service provision instead of asset delivery. This will require a deeper understanding of how financing affects governance and influences tradeoffs, and how project and institutional actors can cultivate shared awareness, knowledge and self-synchronization.

\section{Reflection on Current State}

The original Grand Challenge statements in 2014 set the foundation for the engineering projects community to begin discussing the broader implications of their work and where it fits in the profession and society. With three years of time having passed since that initial statement, and without the formalization available that is presented in this work, a reflection is presented on how that statement has impacted the current work of community members. Specifically, the use of the EPOC 2017 paper topics is used as an indicator for where the community stands today and where it can continue to grow to meet the Grand Challenges. The EPOC 2017 conference papers are used as a reflection point as they represent a snapshot of the topics being addressed by the Engineering Project Organization community. The 67 papers presented at the conference provide an indication of the state of focus on the Grand Challenge topics identified in 2014. Using the five Grand Challenges identified as a basis, Table 2 provides a summary of the number of papers addressing each of the Drivers and the Grand Challenges. 


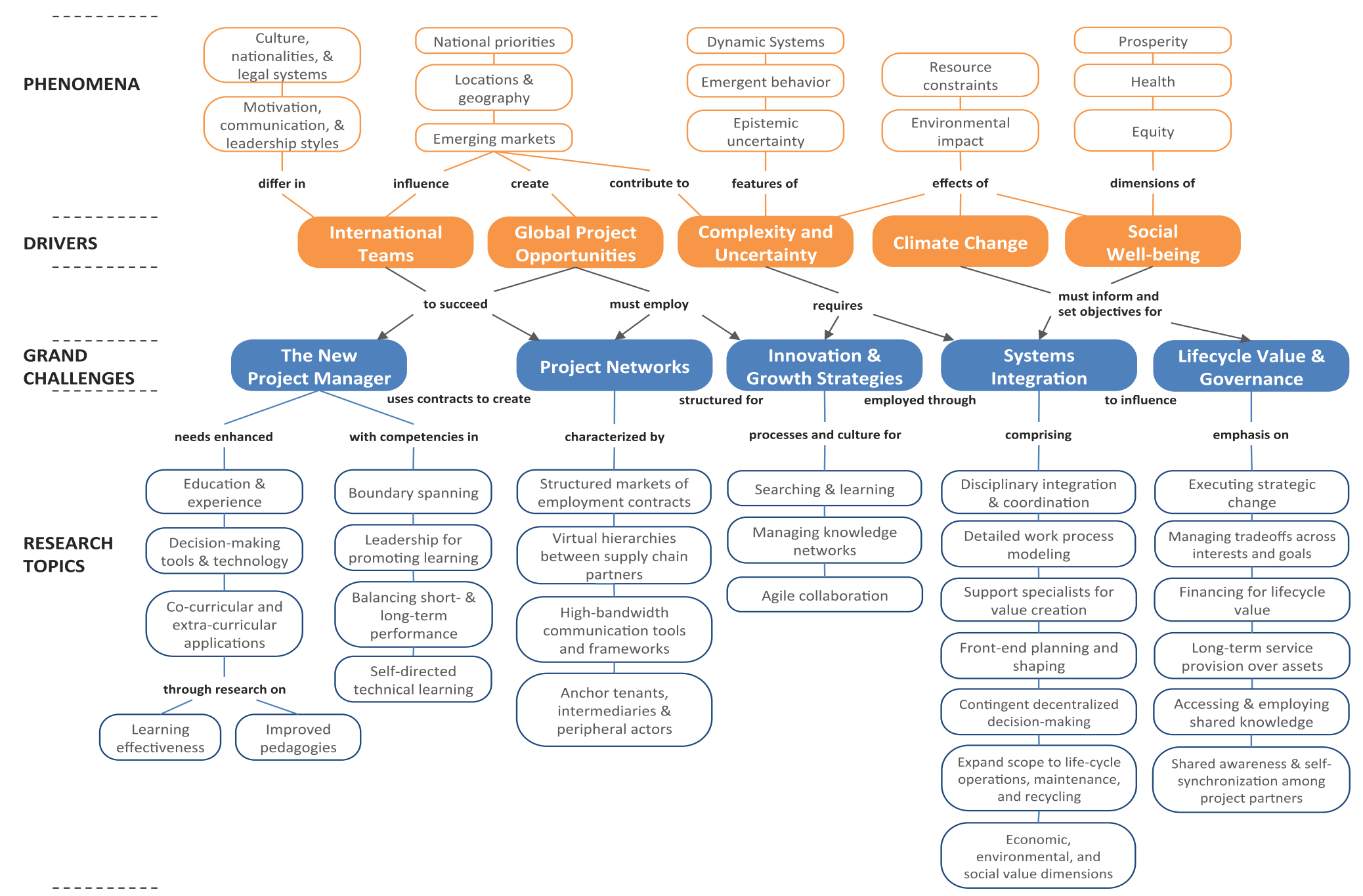

Figure 10. A concept map generated from content analysis of Grand Challenge Vision Statements. Concepts are arranged into two broad categories: 'Drivers' 
As illustrated, the primary Grand Challenge focus is on Lifecycle Value and Governance with the secondary focus on Innovation and Growth Strategies. The three remaining GC areas currently receive little attention in comparison, in the limited context of this conference. Although this is only one snapshot in time, the current state indicates a strong focus on project governance and innovation. Similar to the disproportionate level of attention to a subset of Grand Challenges, the Drivers demonstrate a strong bias towards Complexity and Uncertainty.

\begin{tabular}{|c|c|}
\hline Grand Challenges & Number of Papers \\
\hline $\begin{array}{c}\text { The New Project } \\
\text { Manager }\end{array}$ & 4 \\
\hline Project Networks & 8 \\
\hline $\begin{array}{c}\text { Innovation and Growth } \\
\text { Strategies }\end{array}$ & 14 \\
\hline Systems Integration \\
\hline $\begin{array}{c}\text { Lifecycle Value \& } \\
\text { Governance }\end{array}$ & 30 \\
\hline Drivers & Number of Papers \\
\hline International Teams & 8 \\
\hline $\begin{array}{c}\text { Global Project } \\
\text { Opportunities }\end{array}$ & 4 \\
\hline Complexity and \\
Uncertainty
\end{tabular}

Table 2: The number of EPOC 2017 papers focused on the Drivers and Grand Challenges identified in this synthesis.

From a perspective of reflection, the current topics indicate that there remains a bias towards projectbased research over broader issues within the community. Similarly, the disproportionate focus on the Complexity and Uncertainty driver could be related to the complexity of projects and the interest in addressing this topic as a first-step towards enhancing project delivery. However, both of these disproportionate focal points reflect a movement away from the Drivers that have broader impact in terms of Grand Challenges. Moreover, two GCs - the New Project Manager and Project Networks - uniquely stemmed from the
International Teams and Global Project Opportunities Drivers. A lack of focus on these GCs may be the result of a change in the perceived importance of the Drivers. Views on globalization and cross-cultural collaboration have changed both in academia and industry, as industries move international manufacturing facilities back to their home countries and concerns rise over the impact of offshoring and international work on domestic employment. On reflection, it appears that the Grand Challenges process did not necessarily result in topics that would sustain the interest of the community nor did it have the tangible effect of broadening the community's focus on a greater number of Grand Challenges topics. As a result, the community remains narrowly focused in terms of Drivers and topics. The challenge for the community is thus how to broaden the research agenda to break out of the confines of the more traditional engineering project topics and how to track the evolution of Drivers which change over time.

Achieving this goal of a broader focus on the Grand Challenge issues is challenged by two factors: existing barriers to broadening the research agenda and the need for a specific challenge to the community to achieve greater breadth. In terms of the barriers to broadening an agenda, the principal barrier is the tension between innovation and acceptance. Although academia promotes innovation, acceptance within an academic community remains an underlying concern for any research community. Acceptance being defined either in terms of research grants received and/or papers being accepted for publication in community-accepted journals. From this perspective, the challenge to broaden an agenda to include topics such as 'growth strategies' or 'project networks' is amplified due to the limited number of peers addressing new issues at any given time. The safety in addressing topics that are considered mainstream, fundable, and publishable has a strong influence on any research community. This is reflected in the EPOC 2017 papers that as a group are focused more on the mainstream Challenges and Drivers than the emerging issues.

Additionally, in the category of barriers, there is the barrier of opportunity. Specifically, opportunity to 
explore an emerging area as a $\mathrm{PhD}$ student or an early career faculty. While the focus on doctoral work and early academic career focus is on expanding knowledge through new contributions, there remains the need to find a community where one can pursue new ideas. Given the pressures of acceptance, these opportunities vary across the Grand Challenge topics and the associated Drivers. Moreover, these opportunities emerge and evolve over time as previously discussed. The need to identify, synthesize and disseminate promising research opportunities is arguably the greatest challenge facing the EPO community as creating opportunity also includes risk for the individuals creating them. Balancing this risk with the recognition of the need for broadening the research agenda leads to the need to broaden the charge to the engineering organizations community.

\section{Charge to and Future Directions for the Engineering Project Organization Community}

It has been several years since the Engineering Project Organization community gathered and synthesized the forward looking visionary statements of its most senior community members. Over this time, the Drivers have evolved. How can the Engineering Project Organization community re-envision and re-balance its focus on the Drivers and Grand Challenges? We urge the EPO community to consider several opportunities to achieve this:

1. We have learned through this Grand Challenge visioning process and its implementation that the Drivers creating opportunities for research change and evolve over time. EPO scholars need to consider these Grand Challenges as a living set of critical issues that emerge from these Drivers, and which may emerge, evolve or diminish in importance over time. Hence, the Grand Challenge visioning process should be repeated every five years to identify those new issues that may have emerged and to test the importance of the current set of Grand Challenges. This process needs to be an open-minded and critical one in which the current Grand Challenges are assessed not only by their implementation in the field, but also by their continued importance. It may be, as we have argued in this paper, that a Grand Challenge remains critical but is not receiving adequate attention by scholars. To this end, we are planning a second Grand Challenges summit to take place at EPOC 2019.

2. One way in which these Grand Challenges could be explored in a systematic and comprehensive way, is to have focused journal special issues on each GC. We recommend that calls for papers on corresponding topics and themes be conceptualized and published as Special Issues in related journals and, in particular, in the community's Engineering Project Organization Journal. As a complementary effort, the community could consider refining the Aims and Scope of the Engineering Project Organization Journal to highlight these Grand Challenges and Drivers as the keywords and topic areas of most interest to the community in the near to mid-term. This paper is being published in the inaugural issue of a new version of the Engineering Project Organization Journal and we have explicitly included verbiage related to the Grand Challenges in the Aims and Scope.

3. Invite contributions and hold special topical sessions at the annual EPO conference that focus on specific Grand Challenges and updating those challenges. These sessions can highlight both emergent and significant research by the EPO community in Grand Challenge research areas within the most recent year. To address this, we recommend (1) members of the EPO community that participate in other conferences consider recommending Grand Challenge topics as submission areas in related conferences and (2) that the EPO Conference Committee review the current list of Grand Challenges and plan to include several, if 
not all, of these as invited submission areas.

4. Regardless of the lack of current research emphasis on the New Project Manager Grand Challenge, we believe this topic to be of critical importance and may be one we address in different ways. Many of the EPO community are academics with the dual responsibility of researching Engineering Project Organizations and training the next generation of Engineering Project Organization practitioners and scholars. As such, we recommend, at a minimum, that the academic community include discussion of these Grand Challenges in their pedagogical efforts to teach courses such as Project Management, Project Organizations, Engineering Projects, etc. We also recommend that both the EPO conference and journal consider including a track on EPO pedagogy that may discuss the impact of the New Project Manager Grand Challenge concepts on pedagogy and pedagogical outcomes, among other related topics.

We believe that adopting these recommendations will nudge the EPO community toward a better awareness of the EPO Grand Challenges and to pursue EPO Grand Challenge advances in their research and pedagogy. However, to make the strides necessary to achieve notable advances in the EPO Grand Challenges will require more. We need specific members of the EPO community to champion specific Grand Challenges, to build their research and pedagogical programs around them, to pursue those programs with rigor and passion, and to create the next generation of practitioners and scholars that excel in understanding and improving the field for that specific Grand Challenge. As a community, we need to recognize these emerging Grand Challenge topic leaders and celebrate their accomplishments, perhaps even creating new forms of recognition - such as, EPO Grand Challenge Fellows - to acknowledge the importance of these activities on the health and vitality of the entire EPO community.

Finally, and perhaps most importantly, we must not lose sight of the far reaching impact our work can have on society and the environment provided by this first set of Grand Challenges. At the time, we thought it may be a fundamental shift in our thinking and focus as a scholarly community. However, in our EPOC 2017 paper topic review we observed little focus on research that addresses the Climate Change and Social Well-being Drivers identified in this Grand Challenges elicitation process. It is invigorating to think that our work as a community of scholars on Engineering Project Organization can impact these broad societal issues. We must, as a community, think more about how our work can directly or indirectly address these and other broad societal grand challenges. It is imperative we do our part to have a positive impact on these and other critical issues facing society such as homelessness, poverty, and the need for low-cost housing, the need for low cost, sustainable, and widely available energy, potable water, and sanitation, and the urgent need for healthcare infrastructure globally that equitably serves humanity, among others. We have been provided by this senior and accomplished group of EPO scholars a glimpse into how our work can impact the most pressing issues facing industry, academia, and humanity. It is imperative that we do so.

\section{References}

Baccarini, David. "The concept of project complexitya review." International Journal of Project Management 14, no. 4 (1996): 201-204.

Chi, Cheryl SF, and Raymond Levitt. "Multinational teams incorporating freelance expatriates in the construction industry: case studies of high-speed railways in China and Taiwan." Engineering Project Organization Journal 1, no. 3 (2011): 169-181.

Chinowsky, Paul, Carolyn Hayles, Amy Schweikert, Niko Strzepek, Kenneth Strzepek, and C. Adam Schlosser. "Climate change: comparative impact on developing and developed countries." The Engineering Project Organization Journal 1, no. 1 (2011): 67-80.

Daar, Abdallah S., Peter A. Singer, Deepa Leah Persad, Stig K. Pramming, David R. Matthews, Robert Beaglehole, Alan Bernstein et al. "Grand challenges in chronic non-communicable diseases." Nature 450, no. 7169 (2007): 494-496.

Gann, David, Ammon Salter, Mark Dodgson, and Nelson Phillips. "Inside the world of the project 
baron." MIT Sloan Management Review 53, no. 3 (2012): 63.

Garcia, Rodrigo, Donald Lessard, and Aditya Singh. "Strategic partnering in oil and gas: A capabilities perspective." Energy Strategy Reviews 3 (2014): 2129.

Giles, Jim. "Social science lines up its biggest challenges." Nature News 470.7332 (2011): 18-19.

Goodman, Richard A., Margaret E. Phillips, and S. Sackmann. "The complex culture of international project teams." Modern Organizations and Emerging Conundrums: Exploring the Post Industrial Subculture of the Third Millennium (1999): 23-33.

Gunderson, Goran, Tore, Bjorn, and Raeder, Sabine. "Leading international project teams: The effectiveness of transformational leadership in dynamic work environments," Journal of Leadership \& Organizational Studies, 19.1 (2012): 46-57.

Haberly, Daniel. "Strategic sovereign wealth fund investment and the new alliance capitalism: a network mapping investigation." Environment and Planning A 43, no. 8 (2011): 1833-1852.

Hilbert, David. "Mathematical Problems," (1900).

Hsieh, Hsiu-Fang, and Sarah E. Shannon. "Three approaches to qualitative content analysis." Qualitative Health Research 15.9 (2005): 1277-1288.

Jeuland, Marc, Xun $\mathrm{Wu}$, and Dale Whittington. "Infrastructure development and the economics of cooperation in the Eastern Nile." Water International (2017): 1-21.

Kaminsky, Jessica A., and Amy N. Javernick-Will. "The internal social sustainability of sanitation infrastructure." Environmental Science \& Technology 48, no. 17 (2014): 10028-10035.

Krippendorff, Klaus. Content Analysis: An Introduction to its Methodology. Sage, 2013.

Lessard, Donald, Vivek Sakhrani, and Roger Miller. "House of Project Complexity-understanding complexity in large infrastructure projects." Engineering Project Organization Journal 4, no. 4 (2014): 170-192.
Lousberg, Louis HMJ, Hans WF Wamelink, and Leon IA De Caluwé. "Managing economic feasibility and social relationships: interventions to prevent dysfunctional conflict in public-private partnerships." Engineering Project Organization Journal (2016): 1-10.

Morris, Peter WG, and George H. Hough. The Anatomy of Major Projects: A Study of the Reality of Project Management. Wiley, 1987.

Ramalingam, Shobha, Sunila Lobo, Ashwin Mahalingam, and Jennifer Whyte. "Achieving reliability in transnational work on complex projects: new directions for research." Engineering Project Organization Journal 4, no. 4 (2014): 193-208.

Scott, W. Richard, Raymond E. Levitt, and Ryan J. Orr, eds. Global projects: Institutional and Political Challenges. Cambridge University Press, 2011.

Stinchcombe, A. L. "Contracts as Hierarchical Documents. Organization Theory and Project Management. A. Stinchcombe and CV Heimer. Norwegian University Press, Bergen (Norway) (1985).

Varmus, Harold, et al. "Grand challenges in global health." Science 302.5644 (2003): 398.

Vest, Charles. "Context and challenge for twenty-first century engineering education." Journal of Engineering Education 97.3 (2008): 235-236.

Weber, Robert Philip. Basic Content Analysis. No. 49. Sage, 1990.

Williams, Terry. "Assessing and moving on from the dominant project management discourse in the light of project overruns." IEEE Transactions on Engineering Management 52, no. 4 (2005): 497-508.

White House Office of Science and Technology (OSTP). "2 $1^{\text {st }}$ Century Grand Challenges," (2013). Electronic resource accessed at https://obamawhitehouse.archives.gov/administratio n/eop/ostp/grand-challenges.

Zweig, David, and Bi Jianhai. "China's global hunt for energy." Foreign Affairs (2005): 25-38 